\title{
Detailed Characterization of the Remaining Oil in Matured Water Drive Reservoir
}

\author{
Wang Wenjuan, Lei Xiao, Wang Shichao, Tang Mingguang, Han Xin \\ Research Institute of Western South China Sea Petroleum Administrative Bureau, CNOOC Zhanjiang Branch, Zhanjiang, China \\ Email address: \\ wangwj3@cnooc.com.cn (Wang Wenjuan), leix@cnooc.com.cn (Lei Xiao),wangshzh18@cnooc.com.cn (Wang Shichao), \\ tangmg@enooc.com.cn (Tang Mingguang), hanxin@enooc.com.cn (Han Xin)
}

\section{To cite this article:}

Wang Wenjuan, Lei Xiao, Wang Shichao, Tang Mingguang, Han Xin. Detailed Characterization of the Remaining Oil in Matured Water Drive Reservoir. International Journal of Oil, Gas and Coal Engineering. Vol. 6, No. 1, 2018, pp. 1-7. doi: 10.11648/j.ogce.20180601.11

Received: December 10, 2017; Accepted: December 21, 2017; Published: January 10, 2018

\begin{abstract}
Most of the water-drive oil reservoirs in the western South China Sea had stepped in the middle and high water-cut stage. By the influence of reservoir heterogeneity, fault distribution, well pattern deployment and variation of reservoir flow parameters during long-term natural water drive and water flooding, the remaining oil distribution forecast is not accurate enough, increasing the difficulty of making effective adjustment and potential tapping measures. Through years of tackling key technical problems and field practice, the detailed characterization technique of the remaining oil in matured water drive reservoir was presented. Based on water displacement mechanism, variation of relative permeability curves are derived from Zhang's water-drive characteristic curve during long-term water displacement. In addition, dynamic monitoring data matching was adopted to improve the forecast accuracy of the remaining oil distribution in water flooding oil reservoirs. By combination of flow field, remaining oil saturation field, and remaining oil reserves abundance, comprehensive characterization of water drive dynamic state was realized. The remaining oil enriched areas were quantitatively classified into four levels of potential regions, and corresponding adjustment and potential tapping measures were proposed. This technique had been successfully applied in the middle and high water-cut oilfields in the western South China Sea, with remarkable estimated incremental oil production of approximately $204,000 \mathrm{~m}^{3}$.
\end{abstract}

Keywords: Remaining Oil Distribution, Varying Relative Permeability, Dynamic Monitoring Data Matching, Water Drive Dynamic State

\section{Introduction}

There are 23 natural water drive and water flooding fields in western South China Sea, average water cut has reached $75 \%$. Influenced by reservoir heterogeneity, fault distribution, well pattern deployment and the changes of flow parameters (i.e. permeability, relative permeability, residual oil saturation) after long-term water wash, the contradictions between planes, inter layers and interior layers are obvious. Accurate forecast of remaining oil distribution is difficult due to complicated fluid movement and uneven water drive, arousing the problem of low implementation success ratio of adjustment well in medium-high and high water-cut stages. Effective adjustment and potential exploitation is more and more difficult, the tough challenges mainly consist of the following aspects: (1) Water driving oil mechanism is not well understood. Key parameters affecting seepage flow (i.e. permeability, oil displacement efficiency, rock wettability) changed after long-term water drive [1-6], especially the change of oil displacement efficiency generated appreciable impact on remaining oil distribution and field development efficiency. However, it is difficult to obtain representative oil displacement efficiency with current core displacement experiment method. (2) The numerical simulation of water driving production history is not fine enough. Production logging data or produced water salinity of water flooding reservoir is not considered adequately in traditional oil reservoir numerical simulation. Besides, permeability or relative permeability is not changeable. (3) Characterization of the remaining oil and potential evaluation method in water drive reservoir is unitary. Traditional remaining oil assessment method mainly depends on oil saturation distribution and abundance of remaining reserves, however, overall difference of oil saturation is relatively smaller in the middle and high water-cut stage, so it 
is difficult to accurately divide the potential areas.

A large number of oilfield development practices confirmed that hydrodynamic geological processes are constantly occurring and evolving. Consequently, the parameters of reservoir skeleton, network, seepage, geostress, physicochemical field and flow field are also constantly changing [7]. These changes have further complicated the distribution of remaining oil in high water-cut stage, so it is necessary to carry out detailed characterization of the remaining oil in matured water drive reservoir.

\section{Quantitative Characterization of the Remaining Oil Distribution}

Production history matching is one important segment of oil reservoir numerical simulation, which exerts great impact on prediction result. When water drive reservoir enters the middle and high water-cut stage, fine reservoir numerical simulation needs to integrate geological, experiment data (especially water driving oil mechanism research results), and dynamic monitoring information for comprehensive analysis, hence improving the prediction accuracy of remaining oil distribution.

\subsection{Water Displacing Oil Mechanism}

In the process of long-term water displacement experiment, apart from discontinuous oil drops, aqueous soluble oil is also an important output of oil phase. It is difficult to measure the amount of oil in this displacement process by conventional measurement method, leading to lower calculated oil displacement efficiency. X-CT scanning technique is introduced to solve this problem. Based on the relationship between X-ray intensity and oil content, the saturation distribution along the core and recovery percentage under different multiples of pore volume is calculated. Oil displacement efficiency increased by $8 \%$ in long-term water displacement experiment compared with that acquired by conventional short-term water displacement experiment, which indicates that oil displacement efficiency is changeable during long-term water wash.

Affected by long-term water wash, the porosity, permeability and pore throat structure of water displacement zone apparently changed, the main controlling factor is hydration, expansion, dispersion, and migration of clay mineral (Figure 1). Moreover, the major mechanism of varying oil displacement efficiency is the changing of wettability, capillary number and critical capillary number in the process of long-term water wash [8-12].
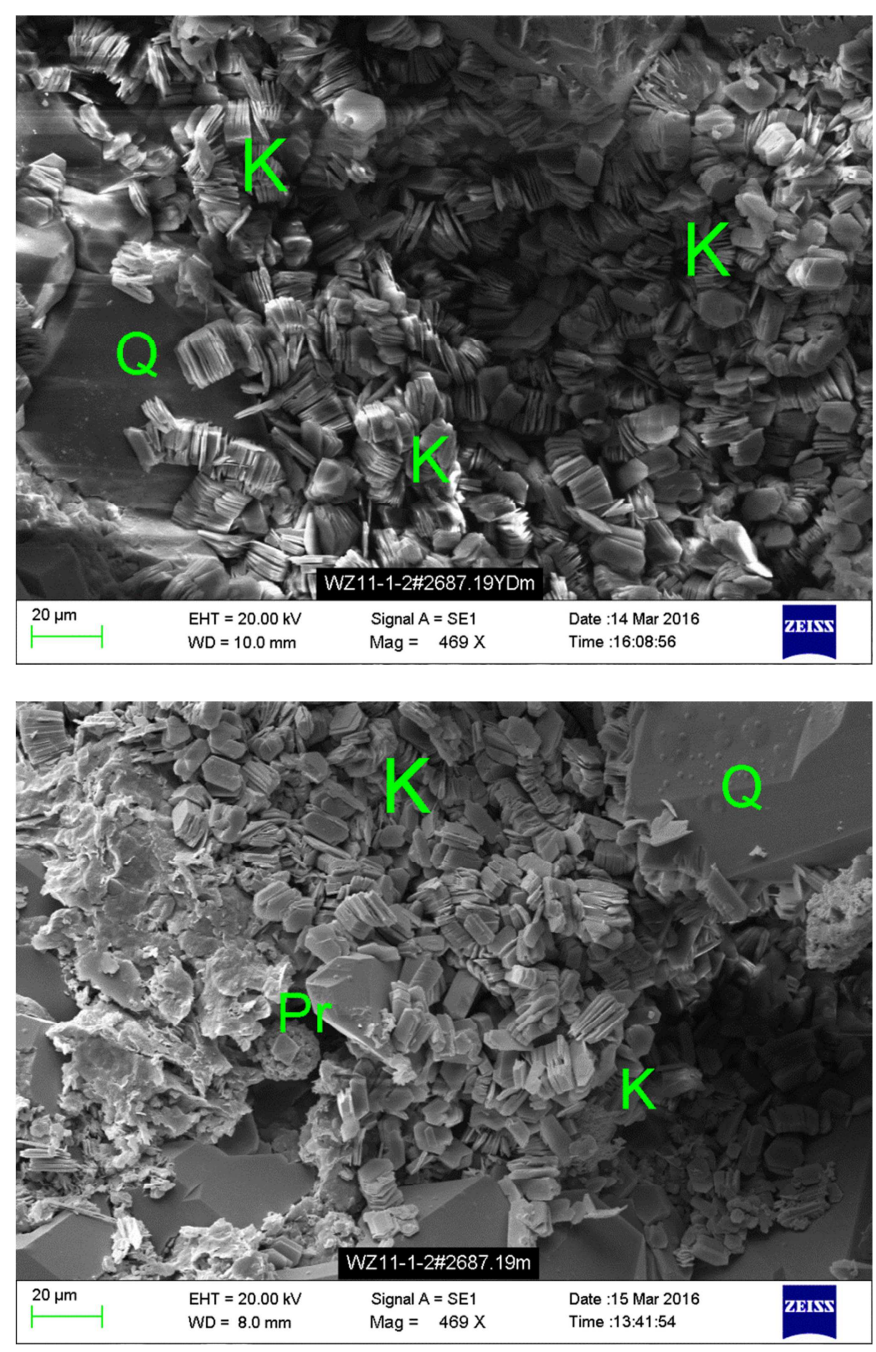

Figure 1. Scanning electron microscope (left: before water wash; right: after water wash). 


\subsection{Variation of Relative Permeability Curves During Long-Term Water Wash}

Influenced by the representativeness of cores and experimental conditions, long-term water displacement experiment results may not necessarily reflect the actual situation underground and the overall dynamic variation rules. Nevertheless, production data is the overall reflection of oil and water movement, so calculation of relative permeability curves using production data is one good way to obtain the variation rule during long-term water wash.

Zhang's water-drive characteristic curve calculated by cumulative oil and water output is simple and easy to use, and its definition is as follows [13]:

$$
\frac{W_{p}}{N_{p}}=a+b \frac{W_{p}}{N_{p}^{2}}
$$

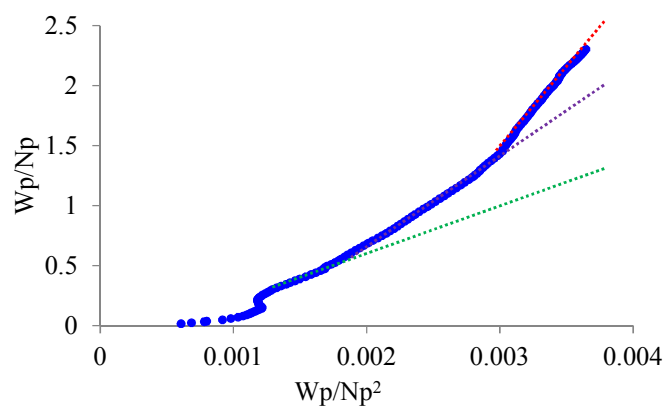

Figure 2. Zhang's water-drive characteristic curve of Formation ZJ2-1U in WC13-2 Oilfield.

Figure 2 shows that there are three obvious straight lines on behalf of three district development stages, indicating that oil and water movement rules changed during 15 years of natural water drive. Conventional practice is using one single straight line to match all the data points, which is apparently unreasonable. Using three different straight lines to match the data points falling in three distinct stages respectively is more appropriate.

Sweep efficiency can be calculated using Zhang's water-drive characteristic curve [14]:

$$
E_{s}=1-\sqrt{\frac{a\left(1-f_{w}\right)}{f_{w}+a\left(1-f_{w}\right)}}
$$

When water cut increases to 1 (100 percent), sweep efficiency also reaches 1 (100 percent). At this moment, the ratio of recoverable reserves to original oil in place (OOIP) is oil displacement efficiency, and then residual oil saturation can be calculated by following equation:

$$
S_{o r}=\left(1-E_{D}\right)\left(1-S_{w c}\right)=\left(1-\frac{b}{N}\right)\left(1-S_{w c}\right)
$$

Recoverable reserves can be obtained by decline analysis of different development stages.

Oil and water relative permeability curves are usually expressed by following empirical formulations:

$$
\begin{gathered}
K_{r o}=K_{r o}\left(S_{w c}\right)\left(\frac{1-S_{o r}-S_{w a}}{1-S_{o r}-S_{w c}}\right)^{m} \\
K_{r w}=K_{r w}\left(S_{o r}\right)\left(\frac{S_{w a}-S_{w c}}{1-S_{o r}-S_{w c}}\right)^{n}
\end{gathered}
$$

Equation (5) divided by Equation (4), and then taking the logarithm, Equation (6) is obtained.

$$
\lg \frac{K_{r o}}{K_{r w}}=m \lg \frac{1-S_{o r}-S_{w a}}{1-S_{o r}-S_{w c}}-n \lg \frac{S_{w a}-S_{w c}}{1-S_{o r}-S_{w c}}+\lg \frac{K_{r o}\left(S_{w c}\right)}{K_{r w}\left(S_{o r}\right)}
$$

Using variable substitution, Equation (6) can be transformed into Equation (7).

$$
\begin{gathered}
y=\lg \frac{K_{r o}}{K_{r w}} \quad x_{1}=\lg \frac{1-S_{o r}-S_{w a}}{1-S_{o r}-S_{w c}} \quad x_{2}=\lg \frac{S_{w a}-S_{w c}}{1-S_{o r}-S_{w c}} \quad t=\lg \frac{K_{r o}\left(S_{w c}\right)}{K_{r w}\left(S_{o r}\right)} \\
y=m x_{1}-n x_{2}+t
\end{gathered}
$$

Parameters of ' $m$ ', ' $n$ ' and ' $t$ ' can be acquired by means of binary linear regression. Taking oil relative permeability at irreducible water saturation as the base permeability, so water relative permeability at residual oil saturation can be expressed as:

$$
K_{r w}\left(S_{o r}\right)=\frac{K_{r o}\left(S_{w c}\right)}{10^{t}}
$$

Assign an initial value to coefficient ' $a$ ', and then adjust its value to match the relationship curve of recovery percentage of OOIP and water cut in each stage, and then the dynamic relative permeability curves are derived (Figure 3 ).

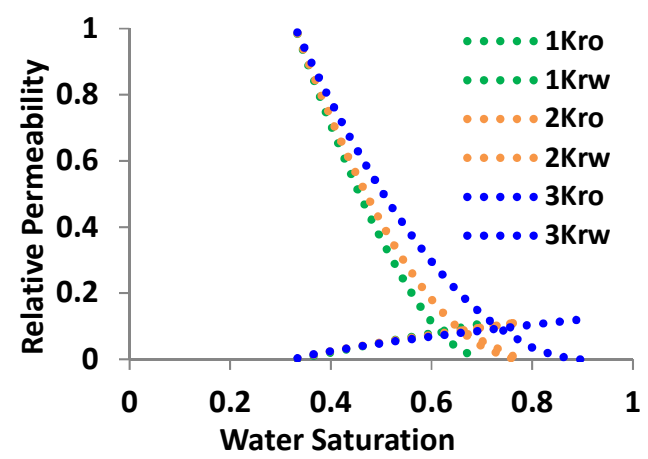

Figure 3. Dynamic relative permeability curves in different development stages.

\subsection{Using Dynamic Monitoring Data to Improve Accuracy of History Matching}

Production logging (PLT \& RPM) data or produced water salinity of water flooding reservoir is not considered adequately in traditional oil reservoir numerical simulation, so it is difficult to guarantee the reasonability of remaining oil distribution for each formation merely matching the water-cut, pressure, GOR and other output data.

Based on water testing analysis data, the type of produced water and the direction of water drive are easier to discriminate by matching produced water salinity (Figure 4). 
In addition, introducing Production logging data will help engineers estimate the rationality of numerical simulation model parameters, thus improving the accuracy of history matching (Figure 5).

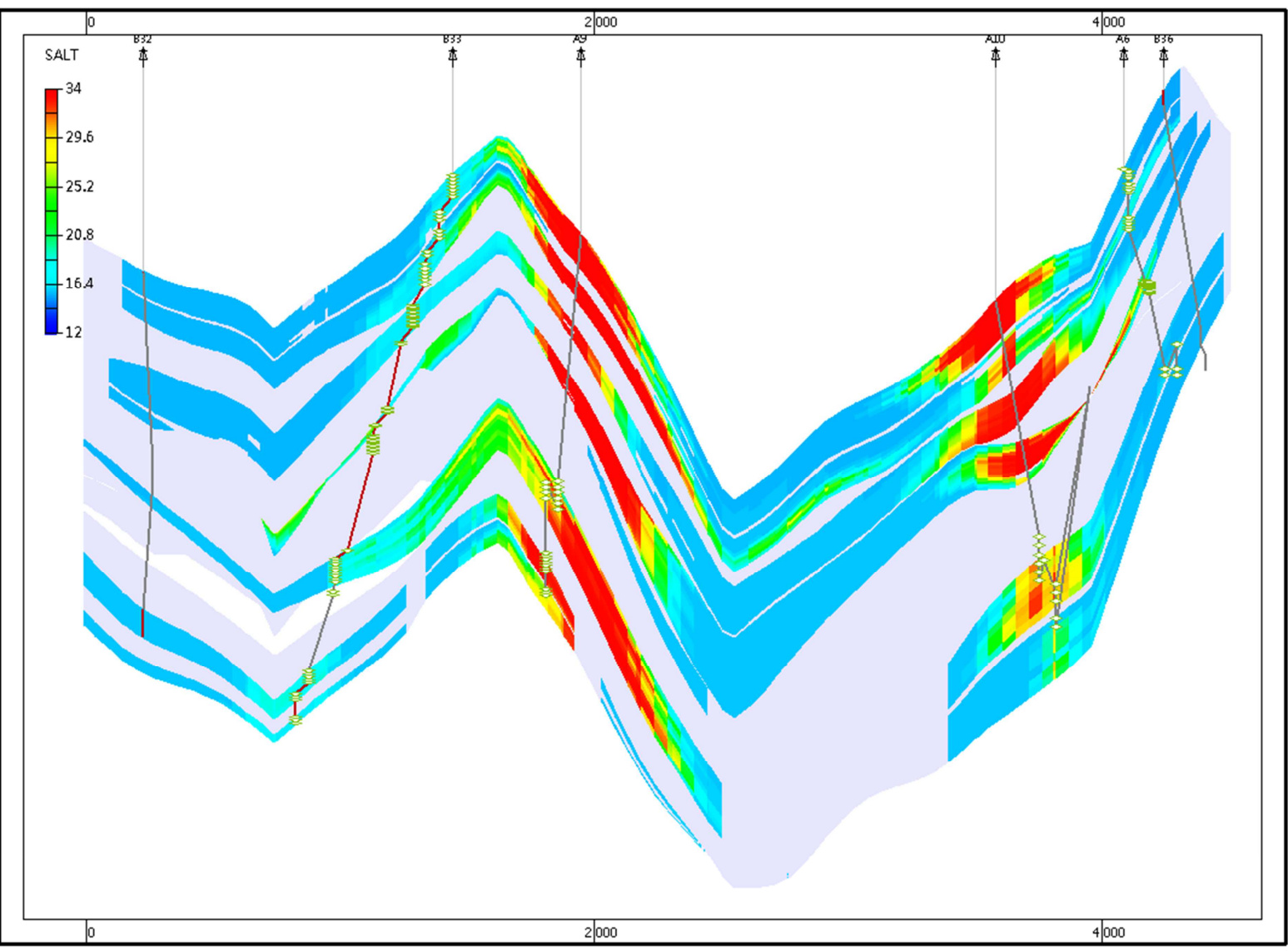

Figure 4. Salinity distribution of Liushagang Formation in WZ11-1 Oilfield.
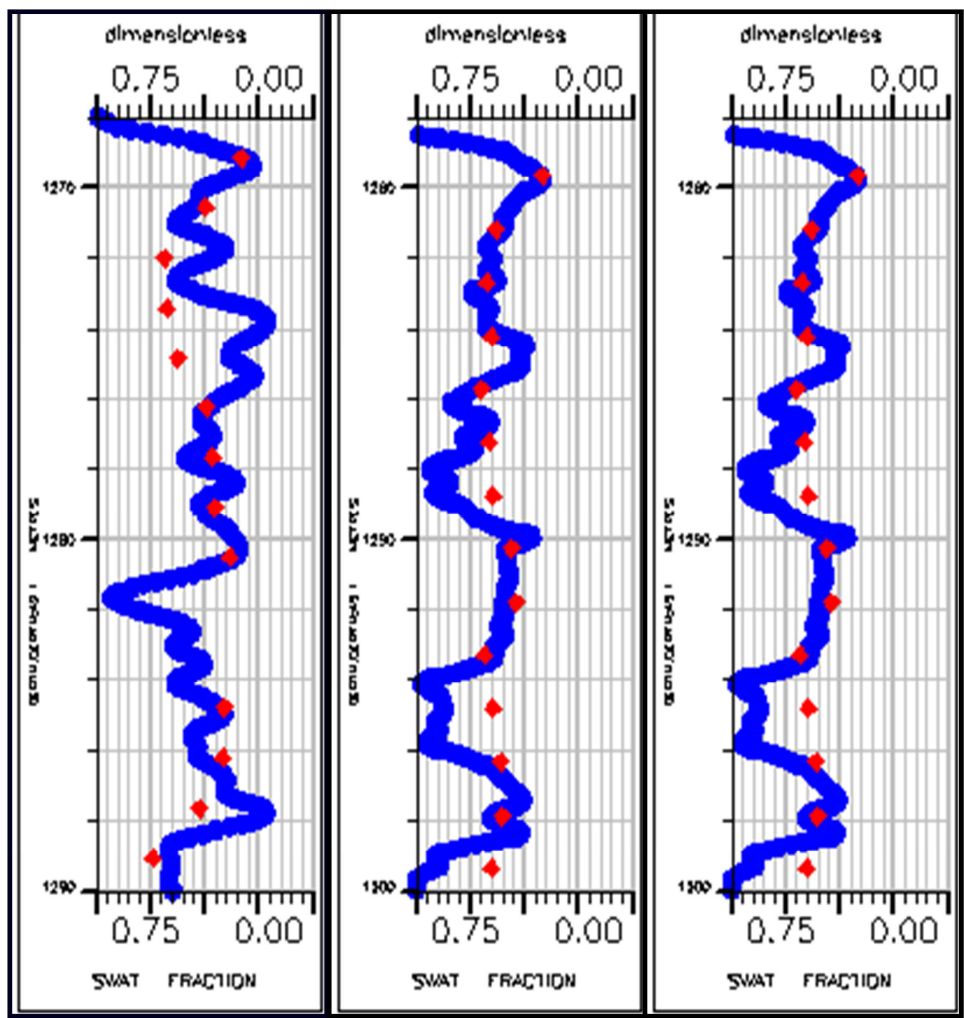

Figure 5. RPM data matching of ZJ2-1U Formation in WC13-2 Oilfield. 


\section{Comprehensive Evaluation of the Remaining Oil Distribution}

Traditional remaining oil assessment method mainly depends on oil saturation distribution and abundance of remaining reserves [15], however, overall difference of oil saturation is relatively smaller in the middle and high water-cut stage, so it is difficult to accurately divide the potential areas.

Flow field distribution is the reflection of displacement levels. Influencing factors of flow field mainly includes two types: static factors and dynamic factors [16]. Static factors consist of sedimentary facies, reservoir heterogeneity, porosity, permeability and fluid viscosity, representing the allowable flowing capability of the reservoir. Dynamic factors consist of fluid velocity, water injection and liquid production, drawdown pressure and surface flux, representing the water wash intensity. By means of logical analysis, surface flux is selected as the sole indicator of flow field. Surface flux is defined as the ratio of cumulative fluid volume to cross-sectional area [17]. According to simulation result, displacing phase (usually water) surface flux can be calculated. Generally, surface flux values are widely distributed, leading to inconvenience of comparison, whereas this problem is well solved by taking the logarithm of surface flux [18].

Flow field is divided into four levels [19]: super, strong, medium and weak (Table 1), and then fluid movement, potential regions of remaining oil (Figure 6) and corresponding adjustment and potential tapping measures (Table 2) are clearly presented. There are tapping potentials in all the three (red, pink and purple) regions if purely considering oil saturation distribution and abundance of remaining reserves, however, the purple region has great implementing risk after observing flow field.

Table 1. Four levels of flow field.

\begin{tabular}{ll}
\hline Flow field intensity & Levels \\
\hline 0 & weak \\
$0-3$ & medium \\
$3-3.9$ & strong \\
$>3.9$ & super \\
\hline
\end{tabular}
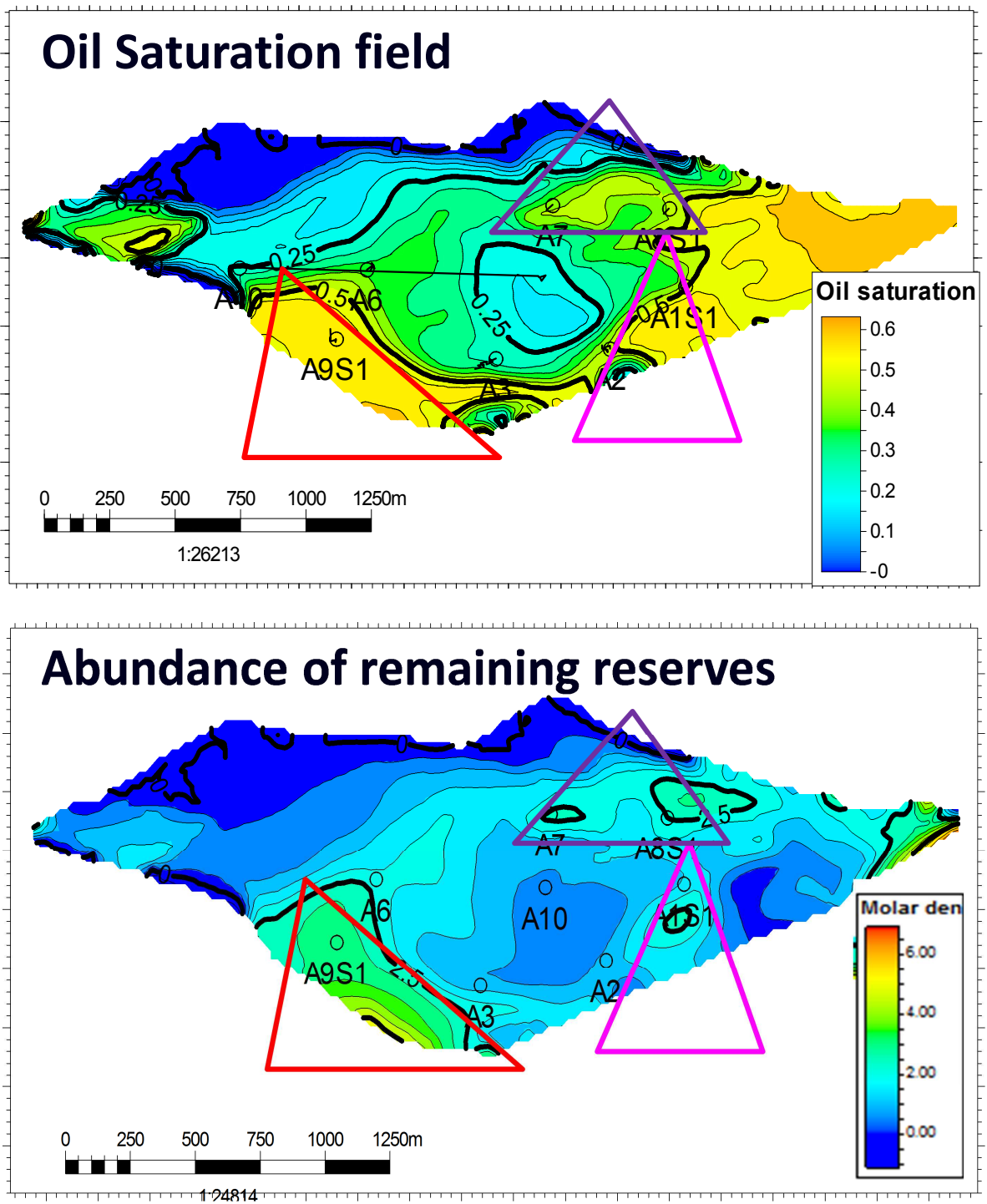


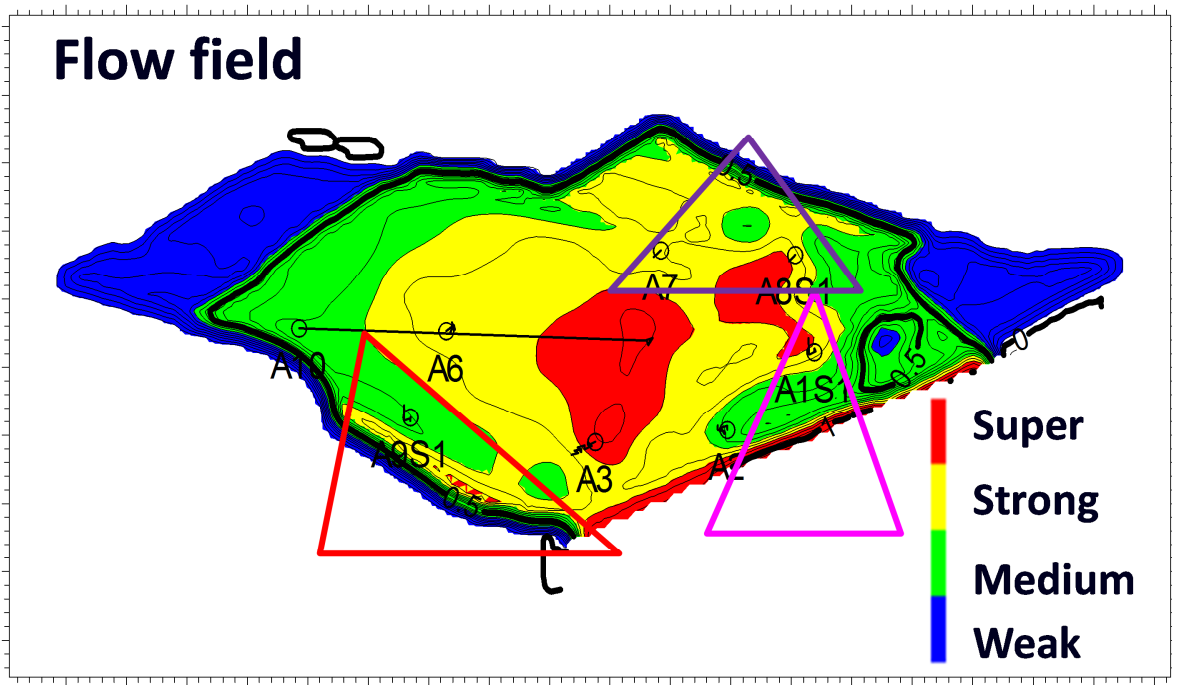

Figure 6. Potential regions of remaining oil obtained by comprehensive evaluation of water drive dynamic state.

Table 2. Grading of potential regions of remaining oil and corresponding adjustment and potential tapping measures.

\begin{tabular}{|c|c|c|c|c|}
\hline Grading & Oil saturation & Flow field & Abundance of remaining reserves & Adjustment and potential tapping measures \\
\hline First Class & Higher & Weak & Higher & Adjustment well \\
\hline Second Class & High & Medium & High & $\begin{array}{l}\text { Increasing liquid production, adjustment well } \\
\text { Increasing liquid production, water shutoff, }\end{array}$ \\
\hline Third Class & Low & Strong & Low & $\begin{array}{l}\text { Shifting producing layer, } \\
\text { Flow field adjustment } \\
\text { Water shutoff, }\end{array}$ \\
\hline Fourth Class & Lower & Super & Lower & $\begin{array}{l}\text { Shifting producing layer, } \\
\text { Conversion of producer }\end{array}$ \\
\hline
\end{tabular}

\section{Field Application}

The technique of detailed characterization of the remaining oil in matured water drive reservoir had been applied over 18 well times in western South China Sea, with adjustment and potential tapping measures of increasing liquid production, adjustment well and optimizing water injection. Up to now, increased oil production has reached $400,000 \mathrm{~m}^{3}$, and estimated ultimate increased oil production will come up to $2,045,500 \mathrm{~m}^{3}$. For example, adjustment well A5S1 was deployed in the first class potential region of WZ11-1 Oilfield, with daily oil output of $101 \mathrm{~m}^{3} / \mathrm{d}$ and water-cut of $5 \%$ at initial production stage. Besides, liquid production rate of $\mathrm{A} 4 \mathrm{H} 1$ and A12H was raised up to $2000 \mathrm{~m}^{3} / \mathrm{d}$ for the third class potential region of WC13-2 Oilfield, with estimated ultimate increased oil production of $96,000 \mathrm{~m}^{3}$.

\section{Conclusions}

Based on the mechanism of water displacing oil, variation of relative permeability curves are derived from Zhang's water-drive characteristic curve. Besides, dynamic monitoring data was adequately used in reservoir numerical simulation, thus improving the accuracy of history matching and remaining oil distribution.

The technique of comprehensive evaluation of remaining oil distribution is presented by combination of flow field, oil saturation field and abundance of remaining reserves. Flow field is divided into four levels, and then grading of potential remaining oil areas and corresponding adjustment and potential tapping measures are proposed.

The technique of detailed characterization of the remaining oil in matured water drive reservoir had been applied in western South China Sea, and estimated ultimate increased oil production will come up to $2,045,500 \mathrm{~m}^{3}$.

\section{Nomenclatures}

$a, b$ : constants got by linear regression;

$N_{\mathrm{p}}$ : cumulative oil production, $10^{4} \mathrm{~m}^{3}$;

$W_{\mathrm{p}}$ : cumulative water production, $10^{4} \mathrm{~m}^{3}$;

$E_{\mathrm{S}}$ : sweep efficiency;

$E_{\mathrm{D}}$ : displacement efficiency;

$f_{\mathrm{w}}$ : water cut;

$K_{\text {ro }}$ : oil relative permeability;

$K_{\mathrm{rw}}$ : water relative permeability;

$S_{\text {wa }}$ : average water saturation;

$S_{\mathrm{wc}}$ : irreducible water saturation;

$S_{\text {or }}$ : residual oil saturation;

$m$ : power of oil relative permeability;

$n$ : power of water relative permeability.

\section{Acknowledgements}

Financial support from the Mechanism and Characterization Method Research of Varying Flow Parameters in Water Drive Reservoirs of China National 
Offshore Oil Corporation (YXKY-2017-ZJ-01) is gratefully acknowledged.

\section{References}

[1] Hu Haiguang. Research on water displacing oil efficiency in extra-high-water-cut stage [D]. Southwest Petroleum University, 2014.

[2] Zhang Chao, Zheng Chuanjiang, Xiao Wu, et al. Research on the influencing factors and functional mechanism of enhanced liquid result in ultra-high water cut stage-case of $7^{4}-8^{1}$ unit of $\mathrm{Es}_{2}$ in the block No. 2 of Shengtuo oilfield [J]. Petroleum Geology and Recovery Efficiency, 2013, 20 (5): 88-91.

[3] Gang Qinlin. New technology for improving water flooding effect of high water-cut oil field [M]. Petroleum Industry Press, 1999.

[4] Wang Qunyi, Bi Yongbin, Xiu Deyan, et al. Research on reservoirs and seepage law in complex fault block oilfield at ultra-high water cut stage [J]. Special Oil \& Gas Reservoirs, 2013, 20 (4): 70-73.

[5] Han Jie, Song Xinmin, Li Jun, et al. Experimental Research on the Changes in Formation Parameters during Water Flooding for Oil Production in Fuyu Oilfield [J]. Science Technology and Engineering, 2013, 13 (14): 3846-3850.

[6] Gao Chao, Yang Manping, Wang Gang. Evaluation of oil/water relative permeability curve feature for water-flooding reservoirs [J]. Complex Hydrocarbon Reservoirs, 2013 (1): 46-49.

[7] Deng Yuzhen. The four dimensional model of reservoir flow field and the study of residual oil: a case study of typical long-term water-flood development in Sheng Li oilfield [M]. Petroleum Industry Press, 2010.

[8] Wu Suying, Sun Guo, Cheng Huiming, et al. Study on the reservoir parameters variation mechanism of sandstone oil reservoir with longterm water drive [M]. Petroleum Geology and Recovery Efficiency, 1999.

[9] Zhu Lihong, Du Qinglong, Li Zhongjiang, et al. Variation of physical characteristics and wettability of the reservoir in high water-cut stage [J]. Petroleum Exploration and Development, 2004, 31 (b11): 82-84.
[10] Yao Tongyu. The Wettability Alteration in Oil Reservoir and Its Effect on Porous Flow Process [D]. Graduate University of the Chinese Academy of Sciences, 2005.

[11] Ji Shuhong, Tian Changbing, Shi Chengfang, et al. New understanding on water-oil displacement efficiency in a high water-cut stage $[\mathrm{J}]$. Petroleum Exploration and Development, 2012, 39 (3): 338-345.

[12] Chen Danqing, Li Jinyi, Zhu Wensen, et al. Experimental research on reservoir parameters variation after water flooding for offshore unconsolidated sandstone heavy oil reservoirs [J]. China Offshore 0il and Gas, 2016, 28 (5): 54-60.

[13] Zhang Jinqing. A new practical water displacement curve [J]. Petroleum Exploration \& Development, 1998 (3): 56-57.

[14] Hu Gang. Improvement and Application of Method for Prediction of Volumetric Sweep Efficiency in Water-Drive Oilfield [J]. Xinjiang Petroleum Geology, 2012 (4): 467-469.

[15] Xie Shiwen, Zhang Wei, Li Qingming, et al. Potential tapping of remaining oil in the late stage of offshore oilfield development based on multidisciplinary integration concept: a case study of $\mathrm{H} 4 \mathrm{C}$ thin reservoir in X3 oilfield, Pearl River Mouth basin [J]. China Offshore Oil and Gas, 2015, 27 (5): 68-75.

[16] Wang Shengdong. Research on Predominant Flow Field and Residual Oil Distribution Considering Gravity Effect [D]. China University of Petroleum, 2007.

[17] Zhang Qiaoliang, Jiang Ruizhong, Jiang Ping, et al. Establishment and Application of Reservoir Flow Field Evaluation System [J]. Petroleum Geology \& Oilfield Development in Daqing, 2014, 33 (3): 86-89.

[18] Lei Xiao, Zhang Qiaoliang, Luo Jihui, et al. Fine characterization technique of remaining oil after water flooding for complex fault block reservoirs in Weizhou oilfields and its application [J]. China Offshore Oil and Gas, 2015, 27 (4): $80-85,92$.

[19] Xin Zhiguo, Jia Junshan, Sun Bo. Research on the Quantitative Determination of the Dominant Flow Field Development Stages [J]. Journal of Southwest Petroleum University: Science \& Technology Edition, 2012, 34 (2): 119-124. 